\title{
A Case of Subcutaneous Myxoid Leiomyosarcoma Misdiagnosed as a Benign Tumor
}

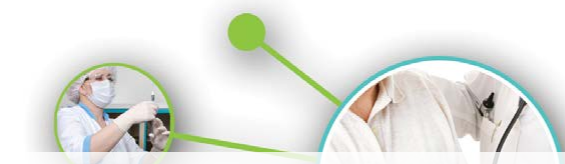

A 46 year old female underwent excision of the thigh mass under general anesthesia with a preoperative sonographic diagnosis of complicated cystic mass. The confirmative diagnosis was a malignant myxoid leiomyosarcoma which was originated in the subcutis. She was treated with a adjuvant radiotherapy after operation and well tolerated without local and distant metastasis at 8 months followup. Even though the mass is small sized and limb located, malignant leiomyosarcoma should be considered.

\section{KEYWORDS: thigh, myxoid leiomyosarcoma, malignant}

\section{Introduction}

Leiomyosarcoma is a rare sarcoma that usually originates in the retroperitoneum, thus often occurring in the uterus. It more rarely originates intramuscularly in the peripheral somatic soft tissue. Myxoid leiomyosarcoma, in which the myxoid stroma occupies more than $50 \%$ of the tissues, is also rarely reported as the myxoid variant of leiomyosarcoma. The previously reported cases of myxoid leiomyosarcoma of the limb originated in the deep soft tissues or intramuscularly, the size varied from 6 to $29 \mathrm{~cm}$, and they were treated through a wide excision, without misdiagnosis. The authors, however, report herein a case with the performance of intraoperative marginal resection under misdiagnosis as a benign complicated cystic mass via ultrasound, and adjuvant radiotherapy was additionally done after the operation. This uncommon malignant tumor, which was misdiagniosed as a benign tumor, is very rarely reported all over the world.

\section{Case Report}

A 46-year-old female patient visited the orthopedic department of the authors' hospital with a palpable lump accompanied by dull pain sustained for nine months on one side of the left thigh. The patient complained of worsening pain and partial heat sense around the tumor with her long-term activities. She insisted that her symptoms were related to an external injury that she suffered when she crashed her thigh against the corner of a desk. Before visiting the authors' hospital, the patient had an ultrasonic inspection and conservative therapy from a local clinic, but her condition did not improve. She was a full-time housewife and had never drank alcohol or smoked but was merely taking a drug for hypertension. She had no special medical condition, except for two Caesarean childbirths 17 and 20 years ago. The physical examinations showed a lump located in the middle and lateral parts of her left thigh, which was round and was $4 \times 5 \mathrm{~cm}$ in size. It was relatively hard and clear but had flexible boundaries. Its skin color could not be distinguished from its surroundings (FIGURE 1). A simple x-ray image showed a circular lump that was radiopaque (FIGURE 2). A lump was found outside the patient's left thigh in the ultrasonic inspection, with an oval to circular shape, heterogeneous separation, a good boundary, and an approximately $3.5 \times 3.2$ $\times 3.6 \mathrm{~cm}$ size. Also, its echogenecity was mixed,

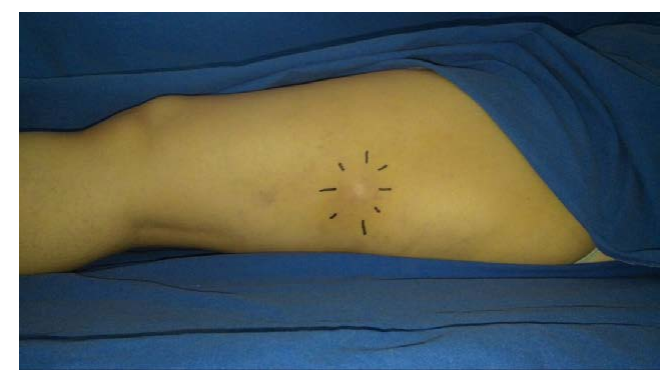

Figure 1: The gross appearance of the mass on the lateral aspect of left thigh was round and relatively hard.

\section{Jihyo Hwang* \\ *Department of Orthopedic Surgery, School of Medicine, Hallym University Chuncheon, Republic of Korea}

*Author for correspondence: hwangjihyo7309@gmail.com 
with high and low echogenicity, and a moderate vascularity was also observed (FIGURE 3). At first, the authors diagnosed the patient's condition as vascularized soft-tissue tumor or a complicated epidermoid cyst via sonography. Moreover, as the tumor was shown to be relatively small (about $4 \mathrm{~cm}$ ) by the ultrasonic inspection, it was not considered a malignant lump. Thus, additional image diagnoses, such as MRI, a bone scan, and biopsy, were not considered, and the patient did not consent to them as well. Lump resection was performed under spinal epidural anesthesia (FIGURE 4) . An approximately $5 \mathrm{~cm}$ longitudinal incision was made on top of the lump, and a round mass was found in the subcutaneous fat layer; marginal resection was thus performed. The lump had a good boundary and was relatively well capsulized, with a $4 \mathrm{~cm}$ size. The transected mass surface was mucoid and slightly bleedy (FIGURE 5). Myxoid leiomyosarcoma was diagnosed from the biopsy after the surgery (FIGURE 6). Additional adjuvant radiation therapy was done after the confirmation of the

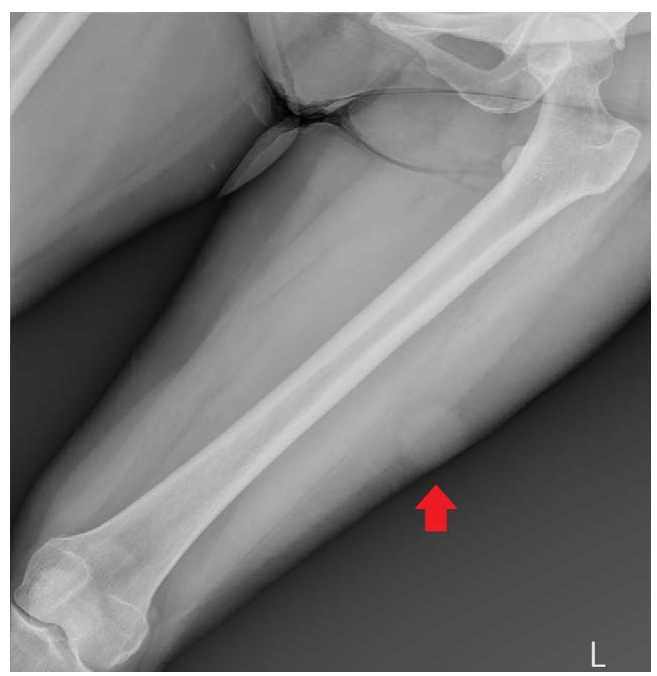

Figure 2: The vague radioopqaue lesion was seen in the plain radiograph (arrow indicated).

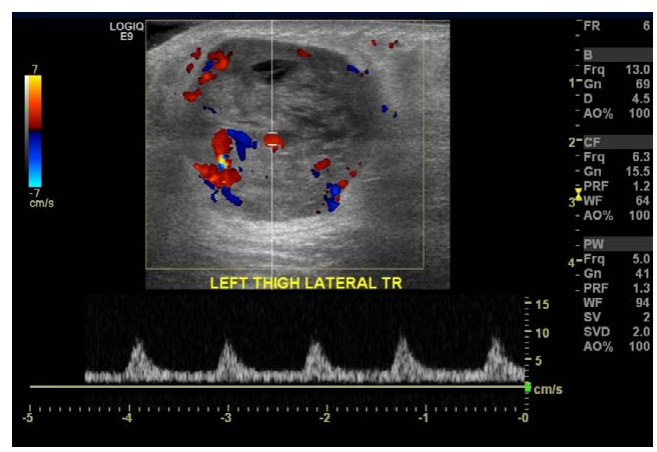

Figure 3: The ultrasonographic image of the mass was $3.5 \times 3.2 \times 3.6 \mathrm{~cm}$ sized, well defined irregular lobulated, round to oval and mixed echogenecity.

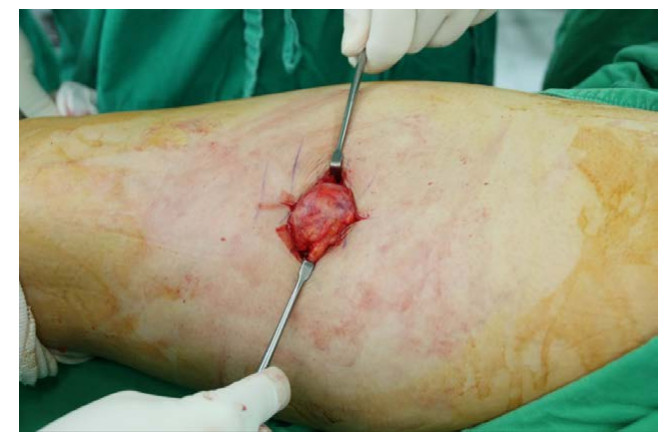

Figure 4: There was a mass at the subcutaneous layer.

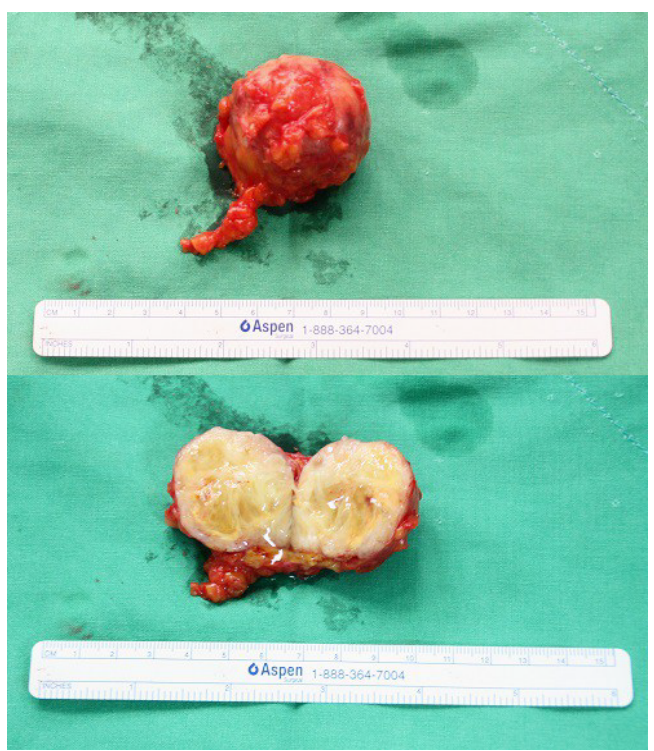

Figure 5: The mass was relatively well capsulated; the center of dissection was lipomatous and myxoid with stippled bleeding.

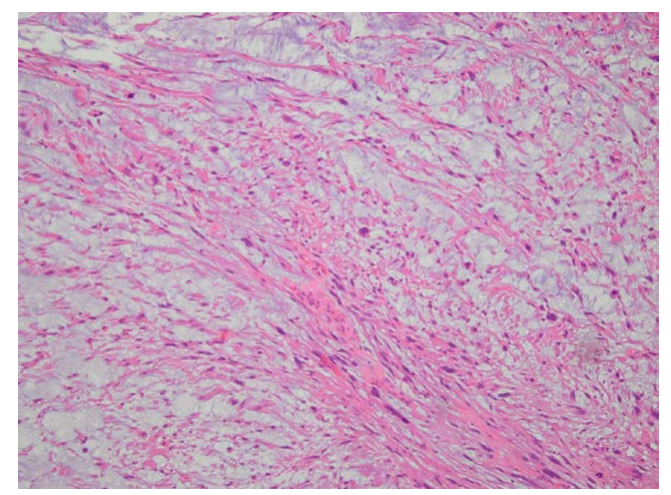

Figure 6: A large amount of myxoid matrix with pleomorphic cellular pattern (hematoxylin and eosin stain, X 100).

diagnosis of malignancy (FIGURE 7). From the observations that were done of the progress of the patient's condition up to the time of the writing of this report, no medical opinion on the condition's recurrence or symptom aggravation has been presented. The patient was informed that data from the case would be submitted for publication, and gave her consent. 


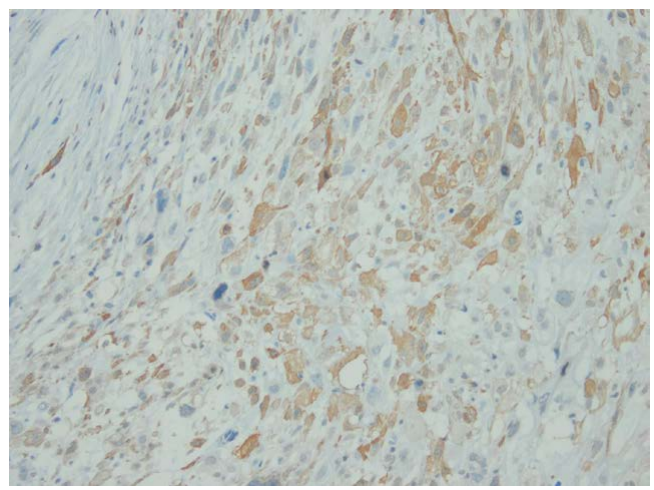

Figure 7: Immunohistochemical staining of smooth muscle cell actin (X 200).

\section{Discussion}

Leiomyosarcoma is an aggressive soft-tissue sarcoma derived from the smooth muscle cells. It is the most common type of sarcoma among the uterus malignancies, of which it accounts for approximately $1 \%$ [1]. Histologically, leiomyosarcoma of the soft tissues is divided into four groups: leiomyosarcoma of the retroperitoneal somatic soft tissues, of cutaneous origin, and of vascular origin, and bone leiomyosarcoma of the somatic soft tissues. Leiomyosarcoma has a number of histologic subtypes, including epithelioid leiomyosarcoma, myxoid leiomyosarcoma, inflammatory leiomyosarcoma, granular cell leiomyosarcoma, and dedifferentiated leiomyosarcoma. Myxoid leiomyosarcoma is extremely rare, with only six cases reported thus far by King et al. 1 in 1982. After this, scientists [2] reported diagnosis guidelines for myxoid leiomyosarcoma, and others [3] published a case report involving a five-year-old female patient in South Korea in 2011. It has since been rarely reported. One of such rare reports is that of Mitrache et al. [4] on a case of uterus myxoid leiomyosarcoma of a 61-year-old female Romanian patient. Case reports of such condition in the liver, spleen, ovary, maxilla, and descending thoracic aorta are even rarer [5-9]. The average affected age is known to be 57.5 years, similar to that for conventional leiomyosarcoma, and $78 \%$ of those affected are female[10]. It is also known that myxoid leiomyosarcoma of cutaneous origin is rarer. There have so far been only two cases of subcutaneous leiomyosarcoma reported by Wile et al. [11], but its histologic subtype was not described. In addition, after the six cases reported by Rubin et al., there has been no report on leiomyosarcoma of the actual myxoid type originating in the limb. Among the aforementioned reported cases, four cases involved the thigh, and two cases involved the foot and the arm. The sizes ranged from 6 to $29 \mathrm{~cm}$, three cases involved the deep soft tissue or muscles, and the others were not mentioned [10]. Therefore, subcutaneous myxoid leiomyosarcoma has not been formally reported anywhere in the world. Also, the wide excision that was done in the previous cases was for therapy purposes, and only one case originating in the arms showed metastasis; the others healed completely. The patient told the authors that the external injury was caused by her hitting her thigh against the corner of a desk, which caused the doctor to confuse the case with a benign tumor such as traumatic granuloma. The tumor was not large enough. There was no medical opinion of malignancy in the results of the ultrasonic inspection before the surgery, and the location was subcutaneous. Under these circumstances, a closer inspection, such as MRI or biopsy, was deemed unnecessary because the tumor's malignancy was doubted due to the aforementioned reasons. Furthermore, based on the fact that myxoid leiomyosarcoma rarely originates in the arms and legs, and that the patient's tumor was almost larger than $5 \mathrm{~cm}$ and was deeply seated, the case reported herein is a very rare case that might be easily misdiagnosed as a benign tumor. Therefore, a closer inspection through biopsy was carefully considered even with the tumor's relatively small size and subcutaneous location, and surgery without biopsy may reduce the need for a re-operation through the wide excision of the tumor, and may eliminate the need for adjuvant treatments after the surgery.

\section{Conclusion}

Myxoid-type leiomyosarcoma is an extremely rare type of tumor. Also, it originates at the subcutaneous level in the arms and legs, and its malignancy may be more difficult to diagnose when it has a small size $(4 \mathrm{~cm})$. Therefore, it is necessary to thoroughly explain the patient's condition to him/her before the operation due to the possibility that malignancy may be detected from the biopsy after the operation. Also, a wide excision may be considered to prevent re-operation. This case of very rare subcutaneous myxoid leiomyosarcoma is hereby reported herein, with a literature review, because it may be easily misdiagnosed as a benign tumor in the realm of orthopedics. 


\section{REFERENCES}

1. King ME, Dickersin GR, Scully RE. Myxoid leiomyosarcoma of the uterus: A report of six cases. The American journal of surgical pathology 6(7):589-598 (1982).

2. Burch D, Tavassoli F, et al. Myxoid leiomyosarcoma of the uterus. Modern Pathology: Lippincott Williams \& Wilkins Philadelphia USA (1998).

3. Park SN, Kim HY, Moon HB. Case Report: A case of Myxoid Leiomyosarcoma of Uterus Occurring in a 5-Year-Old Girl: A case Report and Literature Review. Obstetrics \& Gynecology Science 54(11):712-716 (2011).

4. Mitrache LE, Dumitru VA, Simion
G, Cirstoiu M, Sajin M. A Rare Case of Uterine Myxoid Leiomyosarcoma: a Case Report. MAEDICA-J Clin Med 9(3):269-271 (2014).

5. Lee HJ, Lee JL, Choi WH. A case of primary myxoid leiomyosarcoma of the liver. The Korean journal of internal medicine 17(4):278-282 (2002).

6. Piovanello P, Viola V, Costa G, et al. Locally advanced leiomyosarcoma of the spleen. A case report and review of the literature. World journal of surgical oncology 5(1):135 (2007).

7. Kumar JV, Khurana A, Kaur P, Chuahan AK, Singh S. A rare presentation of primary leiomyosarcoma of ovary in a young woman. ecancermedicalscience. 9(2015).
8. Mizutani H, Tohnai I, Yambe M, UEDA M. Leiomyosarcoma of the maxillary gingiva: a case report. Nagoya journal of medical science 58(3-4):165-170 (1995).

9. Muehlstedt S, Mallery S, Joyce L, Van Camp J. Myxoid leiomyosarcoma of the descending thoracic aorta. The Journal of thoracic and cardiovascular surgery. 121(3):590-592 (2001).

10. Rubin BP, Fletcher CD. Myxoid leiomyosarcoma of soft tissue, an underrecognized variant. The American journal of surgical pathology 24(7):927-936 (2000).

11. Wile AG, Evans HL, Romsdahl MM. Leiomyosarcoma of soft tissue: a clinicopathologic study. Cancer 48(4):1022-1032 (1981). 